\title{
Committee on Publication Ethics 1997-2000
}

\author{
Desmond Johnston
}

Desmond G Johnston PhD FRCP FRCPath FMedSci, Professor of Clinical Endocrinology, Imperial College of Science,

Technology and Medicine, London

Clin Med JRCPL 2001:1:462-3
When clinicians are faced with unethical medical practice, the practitioner may be reported to the General Medical Council (GMC). The GMC investigates, makes a judgement and has the ultimate responsibility to protect the public. The processes involved may be cumbersome and imperfect, but at least they exist. When a journal editor comes across a research paper that he deems to be unethical, the way forward has been less clear. This was the situation until the Committee on Publication Ethics (COPE) was founded in 1997.

COPE is chaired by Michael Farthing and its third report was published in 2000. It was established initially for editors of medical journals from the UK and Europe to sound out colleagues' opinions on possible breaches of research and publication ethics. It may become truly international following discussions with the World Association of Medical Editors. COPE aims to provide a forum for editors and others in medical publishing to discuss relevant issues, encourage ethical standards to be achieved, and to advise on action in cases where such standards appear to have been lowered.

The definition of research misconduct is relatively straightforward when there is a major issue. In most instances, we feel outraged. Examples include:

- drug trials where a new agent is compared with an inactive placebo, when the appropriate comparison would be with an accepted and effective therapy

- new or invasive surgical therapies for conditions where there is no possible rationale for them

- falsified or fabricated data - one researcher in the report is suspected of having fabricated the data for 30 publications.

When this kind of thing is suspected, the host institution should be informed; if the misconduct is confirmed, and if the researcher is a clinician, the GMC should be involved. Studies performed without local ethical approval and proper patient consent should be handled in the same fashion. Duplicate publication is rarely justified and should be considered in the same light. It is the responsibility of the institution where the research is performed to report the investigator to the GMC, and of the GMC to ensure that its actions result in public protection.

The definition of minor research misconduct is more difficult. It includes such misdemeanours as failing to perform a complete literature search before the start of a project, and failure to publish completed work. Most of us will read such sections of the COPE report with a feeling of guilt and relief that we have not been caught out. For many of us, the commonest source of guilt will be the perfectly good studies which have remained unpublished for years because the primary investigator left for another job. One recurring problem, which will induce similar guilt feelings, is the submission and publication of papers where all the authors have not been aware of the final contents. The vitriol with which such papers have been greeted by the 'sleeping' authors in the COPE report has been astounding. In these instances there has often been another agenda, or a major personality issue, but nonetheless most investigators will be more thoughtful about authorship of their own work after reading this report.

COPE provides a forum for discussion and for obtaining consensus but it is not a policing organisation. Editors rely on the host institutions to take action if misdemeanours are suspected. This has not been always the case even in the UK. Reliance is placed on local ethics committees whose performance may not be adequate. Research misdemeanours are committed by non-clinicians as well as by clinicians and there is no equivalent of the GMC for non-clinical scientists. Most journals now are international, and major problems have arisen with some research institutes and national organisations abroad. The researcher (mentioned above) suspected of fabricating the data in 30 publications is the head of his institution and no disciplinary action has yet been, or may ever be, taken. Even if the local institution takes action, and the GMC or equivalent does likewise, there is currently no mechanism to prevent investigators setting up research activities abroad; this has happened on several occasions. As medical science becomes an increasingly international effort, mechanisms to monitor and rectify these failings will become necessary.

The cases in the COPE Report 2000 make interesting reading. A major aspect of this report is its consensus statement. Research misconduct is defined as 'behaviour by a researcher, intentional or not, that falls short of good ethical and scientific standards'. Mechanisms to promote ethical research are outlined. These include: 
- affirming an ethical culture through example

- education throughout people's careers

- vigilance with monitoring and auditing mechanisms

- input to ensure the good performance of ethics committees

- local publication policies

- targeted funding

- mechanisms for investigation of misconduct

- support for whistleblowers.

It has been recommended that a national panel be established to provide advice and assistance. The report is available on the internet (www.publicationethics.org.uk). I commend it to everyone in medical research.
Address for correspondence: Professor D G Johnston, Endocrinology and Metabolic Medicine, Faculty of Medicine, Imperial College of Science, Technology and Medicine, 2nd Floor Mint Wing, St Mary's Hospital, Praed Street, London W2 1NY

The Committee on Publication Ethics held a meeting at the BMA on 15 October 2001 to discuss the question, 'Does the UK need an independent review body to deal with biomedical research misconduct?' Comment on this discussion will be published in Clinical Medicine early in 2002.

Editor 